\title{
High-Performance Forward Osmosis Membranes Used for Treating High-Salinity Oil-Bearing Wastewater
}

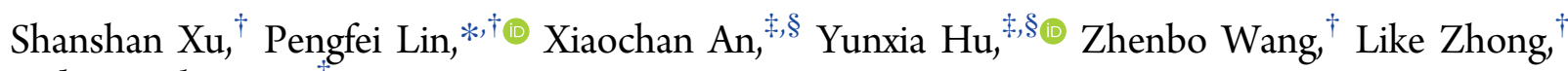 \\ and Qingshan $\mathrm{Niu}^{\dagger}$
}

${ }^{\dagger}$ College of Chemical Engineering, China University of Petroleum (East China), Qingdao, Shandong Province 266580, People’s Republic of China

${ }^{\ddagger}$ State Key Laboratory of Separation Membranes and Membrane Processes, Tianjin Polytechnic University, Tianjin 300387, People’s Republic of China

${ }^{\S}$ CAS Key Laboratory of Coastal Environmental Processes and Ecological Remediation; Yantai Institute of Coastal Zone Research, Chinese Academy of Sciences, Yantai, Shandong Province 264003, People's Republic of China

ABSTRACT: Forward osmosis (FO), which is an emerging osmotic driving membrane separation process, can be directly used in high-salinity oil-bearing (HSOB) wastewater treatment. In this study, the thin film composite (TFC) polyamide FO membranes were fabricated for HSOB wastewater treatment. The impacts of the draw solution (DS) concentration, cross-flow rate, stirring rate, and membrane orientation on membrane performances were researched. The results indicated that the $\mathrm{FO}$ operation mode was better to achieve a stable water flux of TFC membranes as $29 \mathrm{~L} /\left(\mathrm{m}^{2} \mathrm{~h}\right)$ under a cross-flow rate of $15 \mathrm{~cm} / \mathrm{s}$ and $3 \mathrm{M} \mathrm{NaCl}$ as the DS, which was $61 \%$ higher than the water flux in the unoptimized operating conditions $\left(18 \mathrm{~L} /\left(\mathrm{m}^{2} \mathrm{~h}\right)\right)$. The rejection rate of salt and oil was $99 \%$ and $96 \%$. In addition, the effect of different oil concentrations of HSOB wastewater was studied, and the results showed that FO performed well to treat HSOB wastewater when the oil concentration was $<700 \mathrm{mg} / \mathrm{L}$.

\section{INTRODUCTION}

In the process of oil exploitation and refining, a large amount of high-salinity oil-bearing (HSOB) wastewater is produced. ${ }^{1}$ HSOB wastewater has a complex composition, containing a variety of salts, solid particles, dissolved gas, oil slurry, and other organic substances, so the wastewater has high viscosity, high salinity, and is difficult to treat. ${ }^{2,3}$ Discharging HSOB wastewater can not only cause environmental pollution, health hazards, and ecological harm, but also cause waste of water resources and polymers. ${ }^{4}$ In addition, minerals in oilfieldproduced water lead to a decrease in the viscosity of polymer flooding water, which lowers the oil displacement efficiency significantly. ${ }^{5}$ If HSOB wastewater is to be reused, it is necessary to remove the pollutants, especially the minerals. The treatment of HSOB wastewater has become an important issue.

Conventional treatment technologies for HSOB wastewater include hydrocyclone, gas flotation, reverse osmosis (RO), and multieffect evaporation crystallization. ${ }^{1,6}$ However, there were many problems, such as complex equipment, a large footprint, high cost, incomplete treatment, and the secondary pollution of these technologies. ${ }^{7-9}$ Therefore, it is important to find energyefficient, economical, and reliable treatment technologies for HSOB wastewater.

Forward osmosis (FO) is an emerging separation process, which is dependent on the osmotic pressure to drive the water in a feed solution (FS) into a higher concentrated draw solution (DS) through a FO membrane. ${ }^{10}$ The process makes the osmotic pressure difference between the FS and the DS the driving force, which can address several shortcomings of traditional pressure-driven membrane processes, such as high operating pressure, high power consumption, sophisticated membrane modules, and high operation and maintenance costs. ${ }^{11}$ Although FO may not considerably reduce energy consumption, it will be excellent in those scenarios in which $\mathrm{RO}$ is limited, such as highly contaminated wastewater treatment. ${ }^{12}$ FO can be directly applied to the reuse of high salt organic wastewater, advanced treatment of dyeing wastewater, and landfill leachates, which can effectively reduce costs and shorten the treatment process. ${ }^{13-15}$ Compared with RO processes, FO is a green separation process with lower membrane fouling, lower cost of fouling removal, and less chemical cleaning agents. $^{16,17}$ FO can effectively reduce the volume of wastewater in high concentrations and the follow-up treatment costs, especially hybrid FO systems have been used

Received: July 15, 2017

Revised: October 5, 2017

Accepted: October 9, 2017

Published: October 9, 2017 
in oily wastewater treatment with the potential to achieve wastewater zero discharge. . $^{0,13,18}$

In the FO process, the diluted DS can be recovered and utilized via heating, electromagnetic separation, ultrafiltration (UF), nanofiltration (NF), and RO technology, ${ }^{19}$ while the concentrated HSOB wastewater can be treated by evaporation crystallization. ${ }^{18}$ The entire process realized recycling and wastewater zero discharge, which has important environmental protection and economic significance.

In order to efficiently treat HSOB wastewater, this study has focused on the following research: (1) preparing the TFC FO membrane for HSOB wastewater treatment, and evaluating and characterizing the membrane properties, (2) studying the impact of different operating parameters of HSOB wastewater treatment by FO technology to optimize the experimental conditions, and (3) evaluating the effect of FO technology on HSOB wastewater treatment, the rejection rate of oil and salt pollutants, and oil concentration range.

\section{MATERIALS AND METHODS}

2.1. Materials. The chemical agents used throughout this study, such as $\mathrm{NaHCO}_{3}, \mathrm{NaCl}, \mathrm{NaSO}_{4}, \mathrm{~K}_{2} \mathrm{CO}_{3}, \mathrm{CaCl}_{2}$, and $\mathrm{MgCl}_{2}$, were of analytical reagent (AR) grade and purchased from Sinopham Chemical Reagent Co., Ltd., China. Deionized (DI) water (specific resistance, $\geq 10 \mathrm{M} \Omega \mathrm{cm}$ ) was produced in the laboratory. Dimethyl silicone oil was purchased from West Long Chemical Co., Ltd., (China), of which the relative density at $20{ }^{\circ} \mathrm{C}$ was $0.965-0.973 \mathrm{~g} / \mathrm{mL}$ and the viscosity was $330-$ $370 \mathrm{~mm}^{2} / \mathrm{s}$. Emulsifier (Tween 80, chemically pure) was purchased from Sinopham Chemical Reagent Co., Ltd., China. Polysulfone (PSF, Udel P-3500) was purchased from Solvay Advanced Polymers (Brussels, Belgium). N-methyl-2-pyrrolidinone (NMP, anhydrous, 99.5\%), 1,3-phenylenediamine (MPD, $>99 \%$ ), and 1,3,5-benzenetricarbonyl trichloride (TMC, 98\%) were acquired from Sigma-Aldrich (St. Louis, MO, USA). Hexane was obtained from Sinopharm Chemical Reagent Beijing Co., Ltd., (China). All chemicals were used as received.

2.2. Source Water. Based on water quality indicators of polymer-flooding produced water produced from the Daqing oilfield in Heilongiiang Province, which is the biggest oilfield in China, the artificial HSOB wastewater was prepared in the laboratory. The water quality indicators are shown in Table $1 .{ }^{20}$

The artifical water sample was prepared by adding $21.5098 \mathrm{~g}$ of $\mathrm{NaHCO}_{3}, 8.66 \mathrm{~g}$ of $\mathrm{NaCl}, 2.2166 \mathrm{~g}$ of $\mathrm{NaSO}_{4}, 1.1044 \mathrm{~g}$ of $\mathrm{K}_{2} \mathrm{CO}_{3}, 0.7564 \mathrm{~g}$ of $\mathrm{CaCl}_{2}$, and $0.8614 \mathrm{~g}$ of $\mathrm{MgCl}_{2}$ to $8 \mathrm{~L}$ of DI water and mixing well. The degree of mineralization of produced water was simulated. Then, $20 \mathrm{~mL}$ of emulsifier (1 $\mathrm{g} / \mathrm{L}$ ) were added to $124 \mathrm{mg}$ of dimethyl silicone oil and mixed

Table 1. Water Quality Indicators

\begin{tabular}{ll}
\multicolumn{1}{c}{ item } & \multicolumn{1}{c}{ value } \\
$\mathrm{K}^{+}, \mathrm{Na}^{+}$ & $1329.62 \mathrm{mg} / \mathrm{L}$ \\
$\mathrm{Ca}^{2+}$ & $34.07 \mathrm{mg} / \mathrm{L}$ \\
$\mathrm{Mg}^{2+}$ & $17.02 \mathrm{mg} / \mathrm{L}$ \\
$\mathrm{Cl}^{-}$ & $797.85 \mathrm{mg} / \mathrm{L}$ \\
$\mathrm{SO}_{4}{ }^{2-}$ & $187.32 \mathrm{mg} / \mathrm{L}$ \\
$\mathrm{HCO}_{3}{ }^{-}$ & $1952.64 \mathrm{mg} / \mathrm{L}$ \\
$\mathrm{CO}_{3}{ }^{2-}$ & $60.02 \mathrm{mg} / \mathrm{L}$ \\
salinity & $4378.54 \mathrm{mg} / \mathrm{L}$ \\
suspended solids & $15.80 \mathrm{mg} / \mathrm{L}$ \\
oil & $15.50 \mathrm{mg} / \mathrm{L}$
\end{tabular}

well, then gradually added to the above-prepared high-salinity wastewater and stirred at $3500 \mathrm{rpm}$ for $20 \mathrm{~min}$, using a high shearing dispersion emulsifying machine (Model DE-100L, Nantong Clare Mixing Equipment Co., Ltd., China) to prepare HSOB wastewater. ${ }^{21}$ The $\mathrm{pH}$ of the artificial HSOB wastewater was 9.25 .

2.3. Draw Solution. DS is one of the most important factors controlling the FO process, so the selection of an appropriate DS is vital for processing efficiency. A variety of draw solutes have been developed in the past few decades, which are divided into inorganic DS, organic DS, and other $\mathrm{DS}^{22}$ At present, inorganic DS is the most widely used in FO, because of its small molecular weight, high solubility, and high osmotic pressure, which has great potential in HSOB wastewater treatment and wastewater zero discharge. ${ }^{23,24}$

$\mathrm{NaCl}$ solution has high solubility, high osmotic pressure, and is relatively inexpensive. Especially in the coastal areas, seawater can be directly used as DS, which is conducive to the promotion and application of FO technology. Although reverse salt flux of $\mathrm{NaCl} \mathrm{DS}$ is higher, considering the osmotic pressure and cost of the DS, $\mathrm{NaCl}$ solution is selected as the DS for the treatment of $\mathrm{HSOB}$ wastewater in this paper. The diluted $\mathrm{NaCl}$ DS is recovered and utilized by RO technology. A commercially Dow UHP RO membrane was acquired from The Dow Chemical Company (China).

2.4. Fabrication of FO Membranes. FO membranes used in $\mathrm{HSOB}$ wastewater treatment are mainly cellulose triacetate (CTA) membranes and thin film composite (TFC) membranes. Compared with CTA membranes, TFC membranes have higher water flux, lower reverse salt flux, are more selective, and have a wider range of $\mathrm{pH}$, as well as better chemical stability and mechanical strength. ${ }^{15,25,26}$ Considering the membrane materials, process characteristics, and properties of HSOB wastewater, TFC polyamide FO membranes were prepared.

Fabrication of TFC FO membranes involved two steps. First, the PSF support membrane was fabricated by non-solventinduced phase separation (NIPS). Second, a polyamide active layer was formed on the polysulfone support membrane via interfacial polymerization, following the reported procedure. ${ }^{25}$ PSF beads were dissolved in NMP with stirring for $6 \mathrm{~h}$ at room temperature to obtain a $12 \mathrm{wt} \%$ homogeneous casting solution, which was then degassed in a desiccator for at least $12 \mathrm{~h}$. A steel casting knife was used to cast the PSF solution with $150 \mu \mathrm{m}$ gate heights onto the NMP prewetted polyethylene terephthalate (PET) nonwoven fabric. Upon immersion in a water coagulation bath for $10 \mathrm{~min}$ at room temperature, the white PSF support membrane was formed and transferred to DI water for storage. During the casting process, the relative humidity $(\mathrm{RH})$ was set at $40 \% \pm 5 \%$ in a clean room.

To form the polyamide active layer on the PSF support membrane, the top selective side of the wet PSF support membrane was immersed in $3.4 \mathrm{wt} \% \mathrm{MPD}$ aqueous solution for $2 \mathrm{~min}$. Next, the excess MPD was removed from the membrane surface using a rubber roller, the polysulfone support membrane containing MPD was then immersed in a 0.15 wt \% TMC hexane solution for $60 \mathrm{~s}$ to form the polyamide active layer, and cured in DI water at $95{ }^{\circ} \mathrm{C}$ for 120 s. Finally, the TFC membrane was thoroughly rinsed and stored in DI water at $4{ }^{\circ} \mathrm{C}$. Key characteristics of the TFC FO membranes are measured and shown in Table 2.

2.5. Evaluation of the Properties of FO Membranes. A laboratory-scale FO system that was equipped with a cross-flow 
Table 2. Properties of Fabricated TFC FO Membranes

$\begin{array}{lc}\quad \text { property } & \text { value } \\ \text { water permeability, }^{a} A & \left.1.26 \mathrm{~L} /\left(\mathrm{m}^{2} \mathrm{~h} \text { bar }\right)\right] \\ \mathrm{NaCl} \text { permeability, }{ }^{b} \mathrm{~B} & 0.17 \mathrm{~L} /\left(\mathrm{m}^{2} \mathrm{~h}\right) \\ \mathrm{NaCl} \text { rejection, } R^{b} & 98.4 \% \\ \mathrm{pH} & 2-11 \\ \text { thickness } & 92 \mu \mathrm{m}\end{array}$

${ }^{a}$ Measured by RO using DI water as feed solution at a pressure of 13.8 bar (200 psi). ${ }^{b}$ Measured by $\mathrm{RO}$ using $50 \mathrm{mM} \mathrm{NaCl}$ as feed solution at a pressure of $13.8 \mathrm{bar}(200 \mathrm{psi})$.

membrane cell was used to determine water flux and reverse salt flux across the membrane. Two peristaltic pumps (BT6002J, Longer Pump Co., Ltd., China) were used to provide power needed to achieve cycle circulation. The flowchart of the FO membrane setup is shown in Figure 1.

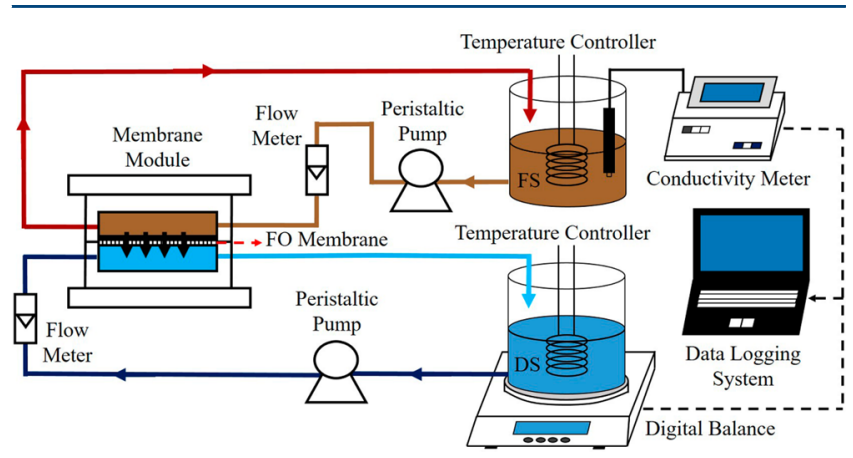

Figure 1. Flowchart of the FO membrane setup.

The cross-flow velocity between the DS and FS was adapted by the peristaltic pump. DI water and HSOB wastewater were used as the FS, and $\mathrm{NaCl}$ solution was used as the DS. Water in the FS was driven into the DS through the FO membrane under the natural osmotic pressure, so that the FS was concentrated and the DS was diluted. The operating temperature was $25{ }^{\circ} \mathrm{C}$, and the operating pressure was atmospheric pressure. The water flux and reverse salt flux was respectively calculated through the detected data of the electronic analytical balance (Model ME203/02, Mettler Toledo Instruments Co., Ltd., Shanghai, China) and electrical conductivity meter (Model DDS-11AW, Shanghai Bante Instruments Limited, China) every $20 \mathrm{~min}$. The oil concentration of the FS and DS was measured by using a total organic carbon (TOC) tester (Model TOC-L, Shimadzu Co., Ltd., Japan) after dilution of the DS 10 times to ensure accurate results under high salinity conditions. Each experiment ran for $120 \mathrm{~min}$ to ensure stable and comparable membrane flux while saving time and operating costs. The experimental process was closed and conformed to the law of conservation of mass. FO membrane was backwashed with DI water for $30 \mathrm{~min}$ after each experiment to keep it clean. Experimental errors were all $<6 \%$.

Water flux (denoted as $J_{\mathrm{w}}\left(\mathrm{L} /\left(\mathrm{m}^{2} \mathrm{~h}\right)\right)$ ) is the water volume through the FO membrane per unit time per unit membrane area, which can be calculated using eq $1:{ }^{27}$

$$
J_{\mathrm{w}}=\frac{\Delta m}{\rho A \Delta t}
$$

where $\Delta m(\mathrm{~g})$ is the weight of water that permeates across the FO membrane in a predetermined time $\Delta t(\mathrm{~h})(20 \mathrm{~min}), \rho$ $\left(\mathrm{kg} / \mathrm{m}^{3}\right)$ is the density of water, and $A\left(\mathrm{~m}^{2}\right)$ is the effective area of the membrane.

Different concentrations of solution on both sides of the membrane resulted in the diffusion of solute from the DS to the FS, which can reduce the FO efficiency. In unit time $t$ and unit effective membrane area $A$, the amount of the solute that diffuses from the DS to the FS is reverse salt flux, which is calculated as follows: ${ }^{27}$

$$
J_{S}=\frac{C_{t} V_{t}-C_{0} V_{0}}{A \Delta t}
$$

where $C_{0}(\mathrm{~mol} / \mathrm{L})$ and $V_{0}(\mathrm{~L})$ are the salt concentration and the volume of the FS at the beginning of the time interval, respectively, while $C_{t}(\mathrm{~mol} / \mathrm{L})$ and $V_{t}(\mathrm{~L})$ are, respectively, the salt concentration and the volume of the FS at time $t$ during the FO tests. $J_{S}\left(\mathrm{~mol} /\left(\mathrm{m}^{2} \mathrm{~h}\right)\right)$ is the reverse salt flux.

The reverse flux selectivity is defined as the ratio of the water flux to the reverse salt flux, which is determined by the membrane active layer and can intuitively characterize the membrane property. ${ }^{28} J_{\mathrm{RFS}}(\mathrm{L} / \mathrm{mol})$ is calculated by eq 3 :

$$
J_{\mathrm{RFS}}=\frac{J_{\mathrm{w}}}{J_{\mathrm{S}}}
$$

The salt rejection rate of the membrane is calculated as follows: ${ }^{29}$

$$
R_{\mathrm{S}}(\%)=\left(1-\frac{J_{\mathrm{S}} / J_{\mathrm{w}}}{C_{\mathrm{DS}}}\right) \times 100
$$

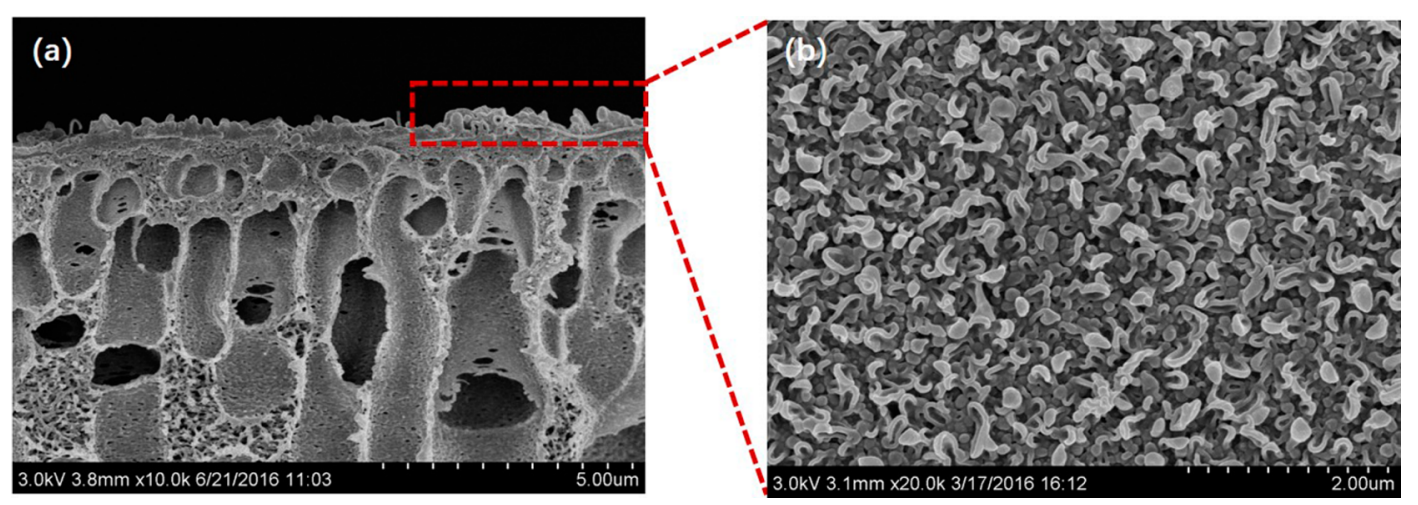

Figure 2. SEM images of the TFC FO membranes: (a) porous layer cross-sectional micrograph and (b) top view of the dense surface layer. 
The oil rejection rate of the membrane can be calculated through the TOC concentration of the FS and DS by eq $5:^{30}$

$$
R_{0}(\%)=\left(1-\frac{C_{\mathrm{s}(t)}}{C_{\mathrm{f}(t)}}\right) \times 100
$$

where $\mathrm{C}_{\mathrm{s}(t)}(\mathrm{mg} / \mathrm{L})$ is the actual permeate concentration of the TOC in the DS at time $t$, and $\mathrm{C}_{\mathrm{f}(t)}(\mathrm{mg} / \mathrm{L})$ is the concentration of the TOC in the FS at time $t$.

\section{RESULTS AND DISCUSSION}

3.1. FO Membrane Performance. 3.1.1. Membrane Characterization. The cross-sectional image and the dense surface layer image of the TFC membranes were detected via scanning electron microscopy (SEM) (Phenom G3, Phenom Scientific Instrument Co., Ltd., Shanghai, China). Membrane samples were dried under vacuum conditions and fractured in liquid nitrogen. Then samples were sputter-coated with gold before SEM imaging. SEM images are shown as Figure 2.

As shown in Figure 2, the TFC FO membrane was composed of a polyamide active layer (AL) formed by interfacial polymerization and a porous ultrafiltration support membrane. The polyamide surface presents a characteristic uniform ridge-and-valley morphology, shown in Figure $2 b$. The porous layer consisted of spongelike pores and fingerlike pores, shown in Figure 2a. The fingerlike pore structure ensured that the membrane had not only high water flux, but also a low degree of internal concentration polarization (ICP). ${ }^{31}$ At the same time, the nonwoven fabric with high toughness and good mechanical properties was used as a support material, and its network structure gave good support for the active layer.

The surface roughness of the TFC membranes was evaluated using atomic force microscopy (AFM) (Model SPM-9700, Shimadzu Co., Ltd., Japan). Membrane samples were dried under vacuum conditions and then smoothly pasted on the AFM. Generally, the ratio of average roughness $\left(R_{a}\right)$ to the root-mean-square roughness $\left(R_{\mathrm{q}}\right)$ represents the membrane surface smoothness. The smaller the values of $R_{\mathrm{a}} / R_{\mathrm{q}}$ that are obtained, the smoother the membrane surface and the better the antifouling property of the membrane. ${ }^{32-34}$ The $R_{\mathrm{a}} / R_{\mathrm{q}}$ value of the TFC membranes was 0.77 , which is less than that of the CTA membranes $\left(R_{\mathrm{a}} / R_{\mathrm{q}}=0.79^{26}\right)$ and the antifouling property of the TFC FO membranes was good.

The contact angle reflects the hydrophilicity of the membrane, and the smaller the contact angle, the stronger the hydrophilicity of the membrane. ${ }^{35}$ The contact angle was measured by the contact angle measurement instrument (Model SD200C, Solon Information Technology Co., Ltd., Shanghai, China). Membrane samples were dried in a vacuum condition and then smoothly pasted on the slide. Ten microliters $(10 \mu \mathrm{L})$ of DI water droplets were deposited dropwise on the membrane surface and the contact angle was observed after $5 \mathrm{~s}$. The experiment was repeated 8 times to get the average value of the contact angle. The average contact angle was $30^{\circ}$, which was $<90^{\circ}$, so the TFC FO membranes had a strong hydrophilic property.

3.1.2. Water Flux and Reverse Salt Flux. The water flux and reverse salt flux of the TFC FO membranes were evaluated using $2 \mathrm{M} \mathrm{NaCl}$ DS under a $15 \mathrm{~cm} / \mathrm{s}$ cross-flow velocity while the AL was directed toward the FS (FO mode). DI water and HSOB wastewater represented the FS, and the oil concentration of HSOB wastewater was $15.5 \mathrm{mg} / \mathrm{L}$. The temperature was maintained at $25^{\circ} \mathrm{C}$ and the pressure was atmospheric pressure. The results are shown as Figure 3.
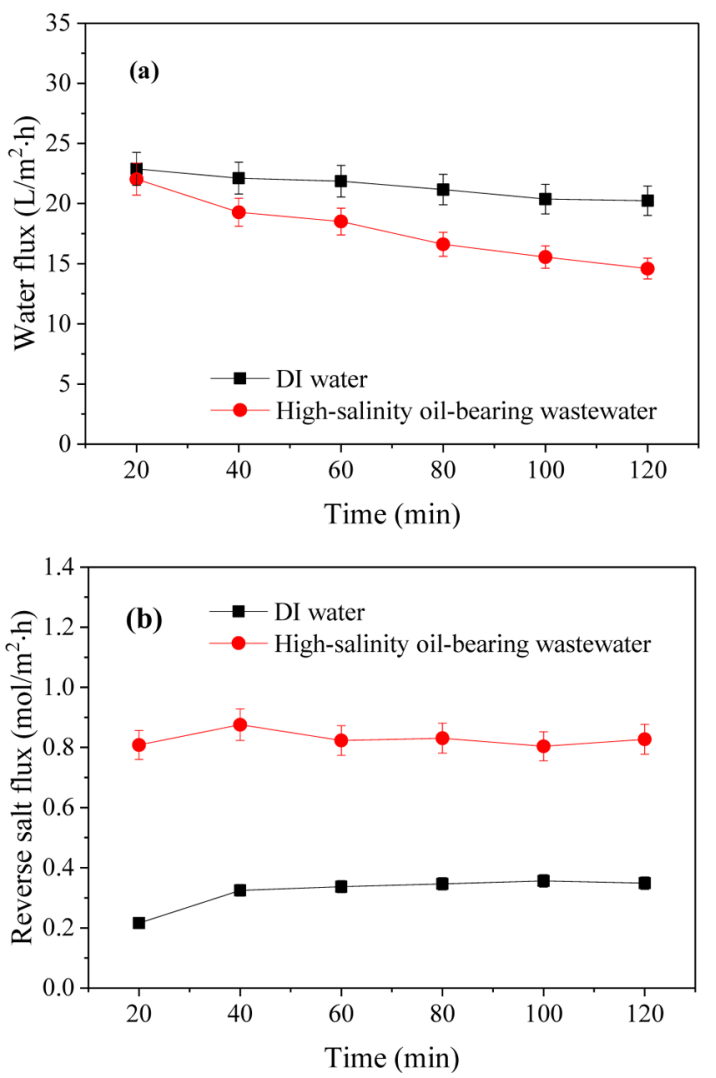

Figure 3. (a) Long-term water flux and (b) reverse salt flux of the TFC FO membranes used for treating $\mathrm{HSOB}$ wastewater $(2 \mathrm{M} \mathrm{NaCl}$ solution as the DS, DI water and HSOB wastewater as the FS, crossflow rate of $15 \mathrm{~cm} / \mathrm{s}$, FO mode, $25^{\circ} \mathrm{C}$ ).

As shown in Figure 3a, both of the water fluxes were reduced, because of the decreased osmotic pressure and the increased concentration polarization during the separation process. The measured average DI water flux was $21 \mathrm{~L} /\left(\mathrm{m}^{2} \mathrm{~h}\right)$, and the average water flux with HSOB wastewater as the FS was $18 \mathrm{~L} /$ $\left(\mathrm{m}^{2} \mathrm{~h}\right)$, which is lower than that observed with the DI water. This was due to the lower osmotic pressure difference needed between the FS and the DS to decrease the driving force and higher boundary layer resistance on the FS side, because of the higher viscosity of HSOB wastewater. ${ }^{36}$ The water flux with HSOB wastewater as the FS decreased faster as the running time increased, which may be attributed to three points. Oil increased the viscosity of HSOB wastewater and the boundary layer resistance, which resulted in feed solute accumulation on the membrane surface during the FO process, and the reverse draw solute also increased the solute concentration on the membrane surface, which was much higher than its concentration in the main body of the FS; this caused more severe external concentration polarization (ECP) ${ }^{37,38}$ Meanwhile, higher reverse salt flux (Figure 3b) led to $\mathrm{Na}^{+}$and $\mathrm{Cl}^{-}$ being more likely to accumulate in the porous layer of the TFC membrane, to exacerbate ICP. ${ }^{26}$ Furthermore, oil and salts in HSOB wastewater were inevitably penetrated into the DS through the membrane. The oil not only caused the organic fouling of the membrane, but also increased the mass-transfer resistance of the membrane, so that the salt ions were easier to 
crystallize around the membrane pores to cause membrane fouling and greatly decreased water flux. ${ }^{39,40}$

The reverse salt flux was measured, and it was found to be higher when HSOB wastewater was used as the FS (Figure $3 b$ ). For the same draw solute, reverse solute flux is mainly proportional to the effective concentration difference on both sides of the membrane active layer, and, theoretically, the effective concentration difference with DI water as the FS is larger than that with HSOB wastewater as the FS. However, the flow direction of water molecules in the FS and solute molecules in the DS in the membrane is reversed. The higher water flux hindered the reverse solute flux, which led to lower reverse salt flux with DI water as the FS. ${ }^{41}$ Furthermore, Arena et $\mathrm{al}^{42}$ and Coday et al. $^{40}$ found that ion rejection decreased with decreasing osmotic pressure for TFC membranes to increase the reverse salt flux, and the presence of cations in the FS would promote the reverse diffusion of the cations in the DS through the TFC FO membranes to increase the reverse salt flux. This may be one of the reasons why HSOB wastewater as the FS has a higher reverse salt flux. The reverse salt flux of both first increased and then remained stable. Since the initial high water flux hindered the reverse salt flux, then both the water flux and the concentration difference between the DS and FS gradually decreased, which resulted in a stable reverse salt flux.

3.2. Optimization of Operating Parameters. The factors that influence the FO membrane properties mainly included the membrane material, membrane structure, and the operating parameters such as the DS, cross-flow velocity, and membrane orientation. ${ }^{43}$ The effects of the concentration of the DS, the cross-flow rate, the stirring rate and the membrane orientation on HSOB wastewater treatment were evaluated to optimize the operating parameters.

3.2.1. Effect of Draw Solution Concentration. The concentration of the DS has an important effect on the water flux. The osmotic pressure determines the driving force in the FO process and is directly related to the concentration of the DS. ${ }^{44}$ Therefore, the effect of different concentrations of the DS on HSOB wastewater treatment was studied to identify the optimum concentration.

HSOB wastewater was used as the FS, and $1 \mathrm{M}, 2 \mathrm{M}, 3 \mathrm{M}$, and $4 \mathrm{M} \mathrm{NaCl}$ solutions were used as the DS in order to study the water flux and reverse salt flux of the FO membrane (i.e., oil concentration of $15.5 \mathrm{mg} / \mathrm{L}$, cross-flow rate of $15 \mathrm{~cm} / \mathrm{s}$, FO mode, $25^{\circ} \mathrm{C}$ ). The results are shown in Figure 4 .

As shown in Figure $4 a$, the water flux decreased with increased time, because of decreased osmotic pressure, increased concentration polarization, and membrane fouling, as described in section 3.1.2. The average water fluxes at the DS concentrations of $1 \mathrm{M}, 2 \mathrm{M}, 3 \mathrm{M}$, and $4 \mathrm{M}$ were $13.72 \mathrm{~L} /\left(\mathrm{m}^{2}\right.$ h), $17.77 \mathrm{~L} /\left(\mathrm{m}^{2} \mathrm{~h}\right), 23.18 \mathrm{~L} /\left(\mathrm{m}^{2} \mathrm{~h}\right)$, and $23.50 \mathrm{~L} /\left(\mathrm{m}^{2} \mathrm{~h}\right)$, the increments of which were $4.05 \mathrm{~L} /\left(\mathrm{m}^{2} \mathrm{~h}\right), 5.41 \mathrm{~L} /\left(\mathrm{m}^{2} \mathrm{~h}\right)$, and $0.32 \mathrm{~L} /\left(\mathrm{m}^{2} \mathrm{~h}\right)$. The water flux increased with increased DS concentration, because of the increased osmotic pressure, which acted as the driving force. However, the water flux increment was greatly reduced when the concentration of the DS was $>3$ M. This was mainly attributed to the increased DS concentration difference that resulted with increased reverse salt flux (Figure 4b), which enhanced the concentration polarization and membrane fouling. ${ }^{45}$ Especially, higher DS concentration accelerated the DS consumption and increased the operating costs. Furthermore, as shown in Figure $4 b$, the increment of the average reverse salt flux was small when the
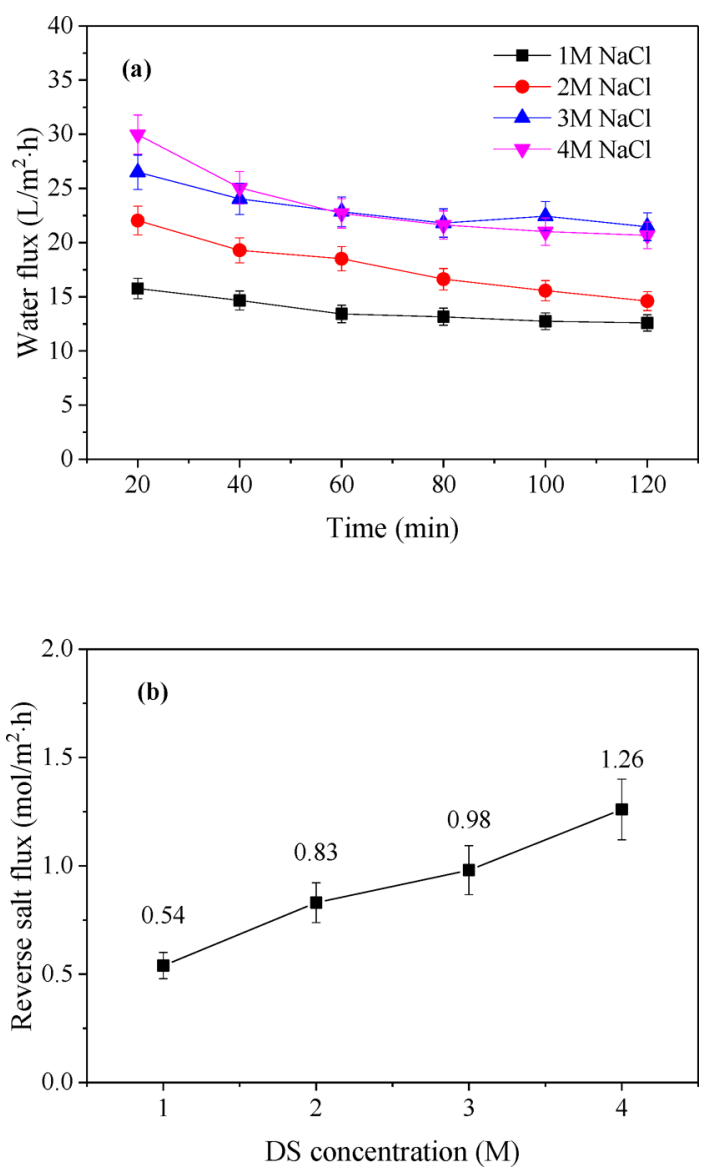

Figure 4. (a) Water flux and (b) reverse salt flux of the TFC FO membranes under different DS concentrations $(1 \mathrm{M}, 2 \mathrm{M}, 3 \mathrm{M}$, and 4 $\mathrm{M} \mathrm{NaCl}$ solutions as the DS, HSOB wastewater as the FS, cross-flow rate of $15 \mathrm{~cm} / \mathrm{s}$, FO mode, $25{ }^{\circ} \mathrm{C}$ ).

concentration of the DS was $3 \mathrm{M}$. This was due to the higher water flux hindering the reverse solute flux, as described in section 3.1.2. Therefore, using a $3 \mathrm{M} \mathrm{NaCl}$ solution as the DS was better.

3.2.2. Effect of the Cross-Flow Velocity. The DS and the FS flow through both sides of the membrane in cross-flow mode. The cross-flow rate influences the water flux of the membrane. Generally, the water flux increases as the cross-flow rate increases in the RO membrane process. ${ }^{44}$ The influence of the cross-flow rate on water flux must be explored further.

HSOB wastewater with $15.5 \mathrm{mg} / \mathrm{L}$ oil concentration as the FS and $2 \mathrm{M} \mathrm{NaCl}$ solution as the FS were applied to investigate the water flux and the reverse flux selectivity of the TFC FO membranes at different cross-flow velocities of the DS and FS (i.e., cross-flow rates of $9-18 \mathrm{~cm} / \mathrm{s}$, FO mode, $25^{\circ} \mathrm{C}$ ). The results are shown in Figure 5.

Figure 5a indicates that the water flux increased with increasing cross-flow velocity, but the growth trend was relatively small. The reason was that the increasing cross-flow rate increased the turbulence at the membrane vicinity, which led to a decrease in the thickness of the ECP layer and an increase of the water flux. However, this phenomenon has shown a strong influence on ion flux but a low influence on volumetric flux. ${ }^{46,47}$ Moreover, the ICP that occurred in the porous support layer was identified to be the main factor for water flux reduction of $\mathrm{FO}$ membranes, which was not effectively reduced by increasing the cross-flow rate, so the 

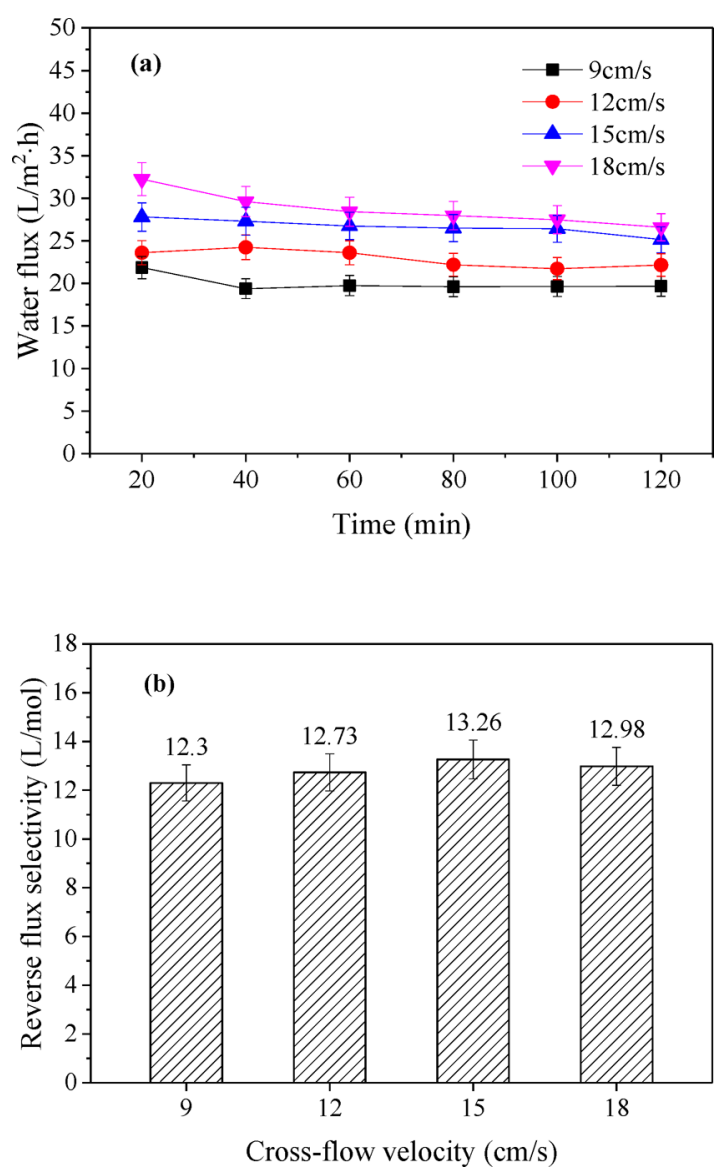

Figure 5. (a) Water flux and (b) the reverse flux selectivity of the TFC FO membranes at different cross-flow velocities of the DS and FS (2 $\mathrm{M} \mathrm{NaCl}$ solution as the DS, HSOB wastewater as the FS, cross-flow rates of $9,12,15$, and $18 \mathrm{~cm} / \mathrm{s}$, FO mode, $25{ }^{\circ} \mathrm{C}$ ).

growth trend of the water flux was relatively small. ${ }^{48}$ In order to further clarify the effect of the cross-flow velocity on the membrane property, the reverse flux selectivity was evaluated (eq 3) as shown in Figure 5b.

As shown in Figure $5 b$, the reverse flux selectivity was different under different flow rates. When the cross-flow velocity was $15 \mathrm{~cm} / \mathrm{s}$, the reverse flux selectivity was the best. This was attributed to the fact that the increased cross-flow rate reduced the ECP of the membrane, which not only increased the water flux but also increased the reverse salt flux. ${ }^{49}$ But the difference in the reverse flux selectivity under different crossflow rates was small, which also indicated that increasing the cross-flow rate could reduce the ECP but had little effect on reducing ICP, even to increase the operating rate. This shows that it is vital to select a suitable cross-flow rate in the FO process.

3.2.3. Effect of the Stirring Rate on the Feed Solution Side. HSOB wastewater has a high viscosity and easily form a boundary layer resistance to reduce water flux in the FO process. It is beneficial to study the effect of the stirring rate on water flux. HSOB wastewater was used as the FS, which was stirred by a digital electric stirrer (Model JJ-1, Changzhou Jintan Liangyou Instrument Co. Ltd., China), and $2 \mathrm{M} \mathrm{NaCl}$ solution was used as the DS to study the water flux of the TFC FO membranes at different stirring rates of $0,100,300$, and 500 rpm on the FS side (i.e., oil concentration of $15.5 \mathrm{mg} / \mathrm{L}$, cross- flow rate of $15 \mathrm{~cm} / \mathrm{s}$, FO mode, $25^{\circ} \mathrm{C}$ ). The results are shown in Figure 6.

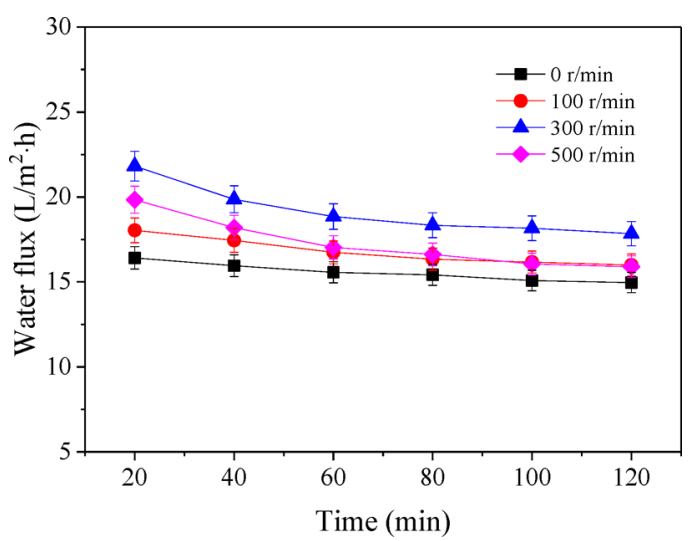

Figure 6. Water flux of the TFC FO membranes at different stirring rates on the $\mathrm{FS}$ side $(2 \mathrm{M} \mathrm{NaCl}$ solution as the $\mathrm{DS}, \mathrm{HSOB}$ wastewater as the FS, stirring rates of $0,100,300$, and $500 \mathrm{rpm}$, cross-flow rate of $15 \mathrm{~cm} / \mathrm{s}$, FO mode, $25^{\circ} \mathrm{C}$ ).

As shown in Figure 6, the average water flux was $15.57 \mathrm{~L} /\left(\mathrm{m}^{2}\right.$ h), $16.79 \mathrm{~L} /\left(\mathrm{m}^{2} \mathrm{~h}\right), 19.14 \mathrm{~L} /\left(\mathrm{m}^{2} \mathrm{~h}\right)$, and $17.78 \mathrm{~L} /\left(\mathrm{m}^{2} \mathrm{~h}\right)$ at the stirring rates of $0,100,300$, and $500 \mathrm{rpm}$. The water flux increased with the increased stirring rate, which was $<300 \mathrm{rpm}$. This was caused by the increased turbulence that enhances the degree of mixing of the FS and reduces the viscosity of HSOB wastewater by stirring the FS, which reduced the boundary layer resistance and decreased the ECP. However, the increase of water flux was not obvious, as described in section 3.2.2. The water flux only increased by $7.83 \%$ at a stirring rate of $100 \mathrm{rpm}$.

However, water flux increased by $22.93 \%$ at a stirring rate of $300 \mathrm{rpm}$, while it decreased rapidly as the time increased. This was attributed to the fact that the higher stirring rate would form a bubbly flow, which could generate intense tangential flow along the membrane and further decrease the ECP, thereby enhancing the water flux. ${ }^{50}$ However, small bubbles may coalesce to form large bubbles in the membrane module, which reduced the effective membrane area and increased the instability of the system, so the water flux reduced rapidly. When the stirring rate increased to $500 \mathrm{rpm}$, the flow was more complicated, which adversely affected water flux and caused higher operating costs. Therefore, it is beneficial to stir the FS with high viscosity and the stirring speed is controlled at $\leq 300$ rpm. However, considering the water flux, the stability of FO system, and the operation cost, the FS may not be stirred in this paper.

3.2.4. Effect of Membrane Orientation. Two different membrane orientations can be applied in the FO process, because of the asymmetric structure of the FO membrane. ${ }^{51}$ The first orientation is the AL toward the FS, which is also known as the FO mode. The second orientation is the AL toward the DS, also known as the pressure-retarded osmosis (PRO) mode. ${ }^{52}$

Since there are many differences in membrane performance between the FO mode and the PRO mode, the results of water flux in both modes were conducted for comparison, as shown in Figure 7. Experimental conditions were as follows: $2 \mathrm{M} \mathrm{NaCl}$ solution as the DS, HSOB wastewater as the FS, oil concentration of $15.5 \mathrm{mg} / \mathrm{L}$, cross-flow rate of $15 \mathrm{~cm} / \mathrm{s}, 25^{\circ} \mathrm{C}$. 


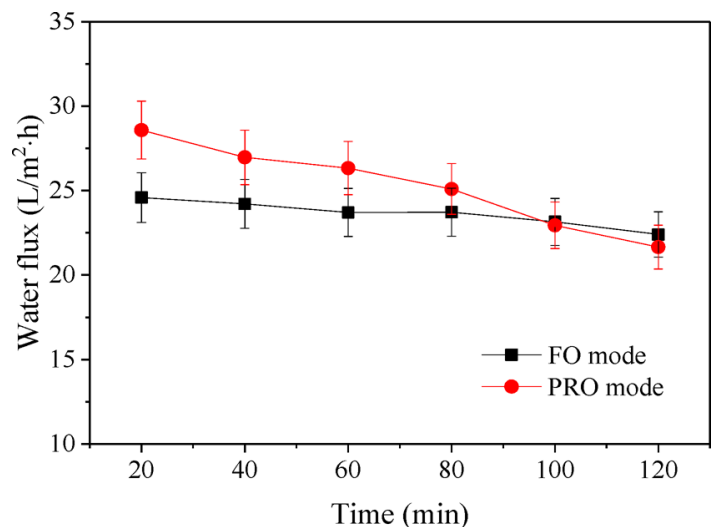

Figure 7. Water flux of the TFC FO membranes under different membrane orientations $(2 \mathrm{M} \mathrm{NaCl}$ solution as the DS, HSOB wastewater as the FS, cross-flow rate of $15 \mathrm{~cm} / \mathrm{s}, 25^{\circ} \mathrm{C}$ ).

Figure 7 shows that the initial water flux in the PRO mode was higher than that in the FO mode. This was due to the fact that the ICP, which could reduce the effective driving force in the FO mode, was more severe than that in the PRO mode. However, the decline of water flux in the PRO mode was faster than that in the FO mode, which was attributed to the selfcompensation of ICP at the draw side in the FO mode. ${ }^{41,53}$ On the other hand, contaminants in the FS readily entered into the porous layer in the PRO mode, to fill the pores and results in flux reduction. Therefore, the stability of the membrane in the FO mode was better. FO membrane orientation has been put forward for the treatment of high fouling feed solutions such as wastewater effluent. ${ }^{54}$ The results are similar to those observed for the treatment of other difficult feed waters in previous FO studies. $^{55-57}$

3.3. Treatment Effect. Using the experimental study on the operating parameters described above, the optimum experimental conditions were established. The water flux and reverse salt flux of the TFC FO membrane under a cross-flow velocity of $15 \mathrm{~cm} / \mathrm{s}$ were evaluated using $3 \mathrm{M} \mathrm{NaCl}$ solution as the DS and HSOB wastewater with an oil concentration of 15.5 $\mathrm{mg} / \mathrm{L}$ as the FS. The membrane orientation was FO mode, the temperature was maintained at $25{ }^{\circ} \mathrm{C}$, and the pressure was atmospheric pressure. Each experiment was operated for 120 min. It is beneficial to stir the FS with high viscosity and the stirring speed is controlled to $\leq 300 \mathrm{rpm}$. The results can provide references for the FO pilot plant test on HSOB wastewater treatment.

The measured water flux could reach $29 \mathrm{~L} /\left(\mathrm{m}^{2} \mathrm{~h}\right)$, which was $61 \%$ higher than the water flux in the nonoptimized operating conditions $\left(18 \mathrm{~L} /\left(\mathrm{m}^{2} \mathrm{~h}\right)\right)$. The reverse salt flux was $1.17 \mathrm{~mol} /$ $\left(\mathrm{m}^{2} \mathrm{~h}\right)$, and the salt rejection rate was $99 \%$, as calculated by eq 4. This indicates that it is important to optimize the operating conditions during the $\mathrm{FO}$ process.

The oil rejection rate of the membrane was evaluated (i.e., 3 $\mathrm{M} \mathrm{NaCl}$ solution as the DS, HSOB wastewater as the FS, crossflow velocity of $15 \mathrm{~cm} / \mathrm{s}$, FO mode, $25{ }^{\circ} \mathrm{C}$, running for 120 $\mathrm{min}$ ) by measuring the content of TOC and the results are shown in Figure 8.

Figure $8 \mathrm{a}$ shows that the TOC concentration of the FS increased after treatment because the FS was more concentrated after the water penetrated to the DS. The TOC concentration of the DS after treatment also increased in Figure $8 \mathrm{~b}$, because some oil entered the DS through the membrane, which inevitably caused contamination to the membrane. The

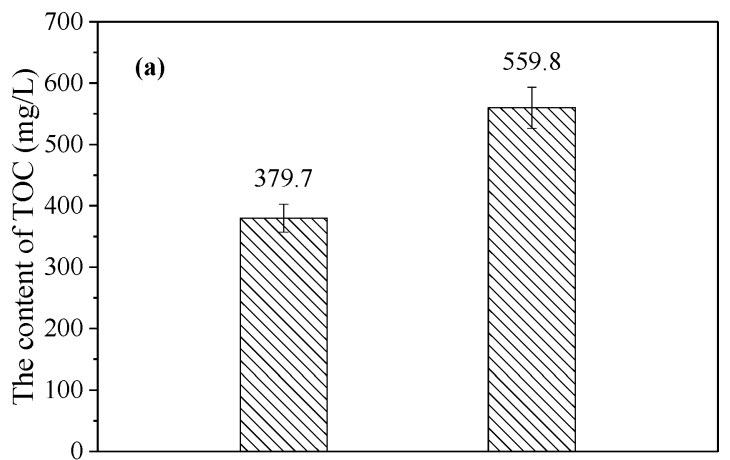

FS before treatment FS after treatment

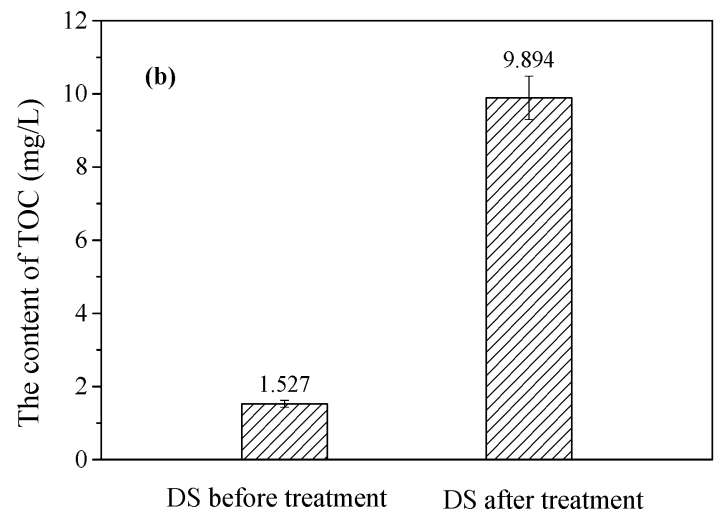

Figure 8. TOC contents of (a) the FS and (b) the DS before and after treatment ( $3 \mathrm{M} \mathrm{NaCl}$ solution as the DS, HSOB wastewater as the FS, cross-flow velocity of $15 \mathrm{~cm} / \mathrm{s}$, FO mode, $25^{\circ} \mathrm{C}$, running for $120 \mathrm{~min}$ ).

rejection rate of oil was $96 \%$ calculated by eq 5 , which showed that the rejection effect of the FO membrane was good.

In order to determine the range of oil concentrations in HSOB wastewater treatment available by the FO technology, as well as to provide a reference for pretreatment, the average water flux in $120 \mathrm{~min}$ was studied in different oil concentrations (from $100 \mathrm{mg} / \mathrm{L}$ to $1300 \mathrm{mg} / \mathrm{L}$ ) under the optimum experimental conditions, and the result are presented in Figure 9.

As shown in Figure 9, the water flux of the FS without oil was the largest while the average water flux decreased as the oil concentration in the FS increased, and the higher the oil

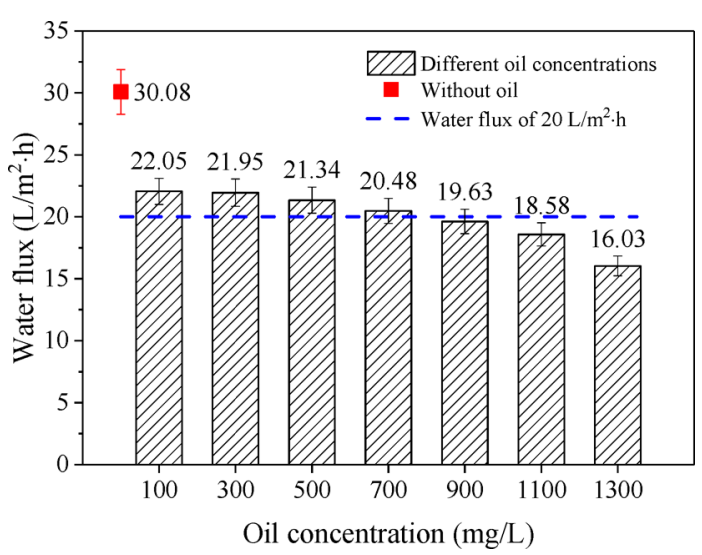

Figure 9. Effect of different oil concentrations in the FS on the average water flux of the TFC FO membranes $(3 \mathrm{M} \mathrm{NaCl}$ solution as the DS, HSOB wastewater in $100-1300 \mathrm{mg} / \mathrm{L}$ oil concentrations as the FS, cross-flow velocity of $15 \mathrm{~cm} / \mathrm{s}$, FO mode, $25^{\circ} \mathrm{C}$, running for $120 \mathrm{~min}$ ). 
concentration, the faster the water flux decreased. The main reason for this phenomenon was that high oil concentrations increased the viscosity of the FS and boundary layer resistance, which led to oil deposition on the membrane surface to cause membrane organic fouling. This increased concentration polarization, blocked the membrane pores, and polluted the membrane. ${ }^{7,58}$ The water flux greatly decreased and $\mathrm{FO}$ will no longer be applicable to treat HSOB wastewater.

In addition, the water flux was more than $20 \mathrm{~L} /\left(\mathrm{m}^{2} \mathrm{~h}\right)$ when the oil concentration was $100-700 \mathrm{mg} / \mathrm{L}$. The oil concentration in oilfield-produced wastewater before entering a water treatment system is generally not more than $500 \mathrm{mg} / \mathrm{L},{ }^{59}$ so it is feasible to treat oilfield produced wastewater with FO technology.

3.4. Membrane Fouling and Recovery. There are many salts and organics in HSOB wastewater, which can cause membrane fouling during the treatment, so it was crucial to study the fouling and recovery properties of the FO membrane. According to the different types of pollutants in membrane fouling, the contaminants can be divided into organic substances, mineral salts, colloidal particles, and microbial species. ${ }^{60}$ The main influencing factors of membrane fouling are solution compositions, membrane materials, hydraulic conditions, and environmental conditions. ${ }^{61}$ However, organic fouling can be effectively controlled by optimizing the hydrodynamic conditions of the FS without chemical cleaning agents in the FO process. ${ }^{16,62}$ Generally, backwashing is utilized to recover the properties of a polluted FO membrane.

Fouling and cleaning experiments were investigated with the FO system described above (Figure 1). HSOB wastewater was treated for $3 \mathrm{~h}$ using $2 \mathrm{M} \mathrm{NaCl} \mathrm{DS}$ at a cross-flow velocity of 15 $\mathrm{cm} / \mathrm{s}$. The membrane then was subjected to physical cleaning for $30 \mathrm{~min}$ using $5 \%$ of the recovered water $(\sim 100 \mathrm{~mL}$ pure water) on both sides of the membrane at a reverse cross-flow velocity of $24 \mathrm{~cm} / \mathrm{s}$. The results showed that the average water flux of the FO membrane after cleaning reached 99\%, compared with a new membrane, as shown in Figure 10.

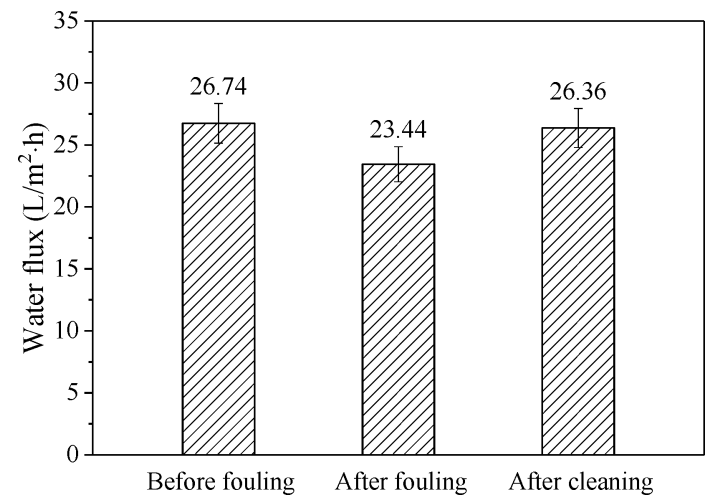

Figure 10. Average water flux of the TFC FO membranes before fouling, after fouling, and after cleaning in $\mathrm{FO}$ mode $(2 \mathrm{M} \mathrm{NaCl}$ solution as the DS, HSOB wastewater as the FS, backwashing velocity of $24 \mathrm{~cm} / \mathrm{s}$, backwashing time of $30 \mathrm{~min}$, FO mode, $25^{\circ} \mathrm{C}$ ).

This was attributed to the strong hydrophilic nature of the TFC FO membranes, hydrophobic oil, and natural osmotic pressure as a driving force in FO, which resulted to contaminants accumulated on the membrane surface to form a loose and sparse fouling layer. The fouling layer can be washed away by simple cross-flow cleaning.

\section{CONCLUSIONS}

This study focused on the feasibility and effectiveness analysis of HSOB wastewater treatment by the FO membrane. Considering membrane materials, process characteristics and properties of $\mathrm{HSOB}$ wastewater, the TFC polyamide FO membranes were fabricated. The factors that influence the FO membrane properties on HSOB wastewater treatment then were evaluated and the optimized experimental conditions were determined. The cross-flow velocity was $15 \mathrm{~cm} / \mathrm{s}$, and $3 \mathrm{M}$ $\mathrm{NaCl}$ solution was chosen as the DS. The FO mode was better to achieve a stable water flux. Temperature was $25^{\circ} \mathrm{C}$, and the pressure was atmospheric pressure.

The measured water flux could reach $29 \mathrm{~L} /\left(\mathrm{m}^{2} \mathrm{~h}\right)$, which was $61 \%$ higher than the water flux in the unoptimized operating conditions $\left(18 \mathrm{~L} /\left(\mathrm{m}^{2} \mathrm{~h}\right)\right)$. The rejection rates of salt and oil were $99 \%$ and $96 \%$, respectively. When the oil concentration was $100-700 \mathrm{mg} / \mathrm{L}$, the separation method of the FO membrane was utilized to treat HSOB wastewater with good effect. The oil concentration in the oilfield produced wastewater is not more than $500 \mathrm{mg} / \mathrm{L}$, so it is feasible to treat the oilfield produced wastewater from polymer flooding with FO technology.

\section{AUTHOR INFORMATION}

\section{Corresponding Author}

*Tel.: +86-532-86983481. Fax: +86-532-86981815. E-mail: lpf@upc.edu.cn.

\section{ORCID}

Pengfei Lin: 0000-0002-2403-6767

Yunxia Hu: 0000-0003-4710-6542

\section{Notes}

The authors declare no competing financial interest.

\section{ACKNOWLEDGMENTS}

This study was supported by the Fundamental Research Funds for the Central Universities (No. 15CX02016A), the Open Project of State Key Joint Laboratory of Environmental Simulation and Pollution Control (Tsinghua University) and the Science and Technology Planning Project of Qingdao (No. 15-9-2-111 nsh). The authors thank Dr. Edward C. Mignot (Shandong University), for linguistic advice.

\section{REFERENCES}

(1) Yu, L.; Han, M.; He, F. A review of treating oily wastewater. Arabian J. Chem. 2017, 10, S1913-S1922.

(2) Jamaly, S.; Giwa, A.; Hasan, S. W. Recent improvements in oily wastewater treatment: Progress, challenges, and future opportunities. J. Environ. Sci. 2015, 37, 15-30.

(3) Alzahrani, S.; Mohammad, A. W. Challenges and trends in membrane technology implementation for produced water treatment: A review. J. Water Process Eng. 2014, 4, 107-133.

(4) Ye, C.; Chen, C.; Zhou, W. Progress of the treatment technology of oilfield wastewater. Mod. Chem. Ind. 2015, 35 (3), 55-58 (in Chin.).

(5) Jing, G.; Xing, L.; Liu, Y.; Du, W.; Han, C. Development of a four-grade and four-segment electrodialysis setup for desalination of polymer-flooding produced water. Desalination 2010, 264 (3), 214219.

(6) Liang, W.; Zhao, X.; Han, Y.; Qiu, G. Discussion on the treatment of wastewater containing polymers in oilfields and its utilization. Ind. Water Treat. 2010, 30 (10), 1-5 (in Chin.).

(7) Liu, K. The application study of an effective hollow fiber membrane in oily waste water separation. M.S. Thesis, Suzhou 
University of Science and Technology, Suzhou, China, 2015 (in Chin.).

(8) Saththasivam, J.; Loganathan, K.; Sarp, S. An overview of oilwater separation using gas flotation systems. Chemosphere 2016, 144, 671-680.

(9) Munirasu, S.; Haija, M. A.; Banat, F. Use of membrane technology for oil field and refinery produced water treatment-A review. Process Saf. Environ. Prot. 2016, 100, 183-202.

(10) Chekli, L.; Phuntsho, S.; Kim, J. E.; Kim, J.; Choi, J. Y.; Choi, J.; Kim, S.; Kim, J. H.; Hong, S.; Sohn, J.; Shon, H. K. A comprehensive review of hybrid forward osmosis systems: Performance, applications and future prospects. J. Membr. Sci. 2016, 497, 430-449.

(11) McCutcheon, J. R.; Elimelech, M. Influence of concentrative and dilutive internal concentration polarization on flux behavior in forward osmosis. J. Membr. Sci. 2006, 284 (1-2), 237-247.

(12) Shaffer, D. L.; Werber, J. R.; Jaramillo, H.; Lin, S.; Elimelech, M. Forward osmosis: Where are we now? Desalination 2015, 356, 271284.

(13) Zhang, S.; Wang, P.; Fu, X.; Chung, T. Sustainable water recovery from oily wastewater via forward osmosis-membrane distillation (FO-MD). Water Res. 2014, 52, 112-121.

(14) Ge, Q.; Wang, P.; Wan, C.; Chung, T. Polyelectrolyte-promoted forward osmosis-membrane distillation (FO-MD) hybrid process for dye wastewater treatment. Environ. Sci. Technol. 2012, 46 (11), 62366243.

(15) Cath, T.; Childress, A.; Elimelech, M. Forward osmosis: Principles, applications, and recent developments. J. Membr. Sci. 2006, 281 (1-2), 70-87.

(16) Lee, S.; Boo, C.; Elimelech, M.; Hong, S. Comparison of fouling behavior in forward osmosis (FO) and reverse osmosis (RO). J. Membr. Sci. 2010, 365 (1-2), 34-39.

(17) Mi, B.; Elimelech, M. Organic fouling of forward osmosis membranes: Fouling reversibility and cleaning without chemical reagents. J. Membr. Sci. 2010, 348 (1-2), 337-345.

(18) Shao, G.; Fang, D. Application of MBC zero liquid discharge technology to desulfurization wastewater treatment in a power plant. Ind. Water Treat. 2016, 36 (8), 109-112 (in Chin.).

(19) Li, Q.; Ma, W.; Nie, C.; Yan, Y. Draw solution for forward osmosis processes: Types, separation and recovery process. Water Pollut. Control 2016, 34 (3), 11-17 (in Chin.).

(20) Xu, J.; Yu, S.; Sun, Y.; Pang, H.; Liang, C.; Yang, X. Ultrafiltration-experimental research on technology for desalination of polyvinylidene fluoride. J. Harbin Univ. Commerce (Nat. Sci. Ed.) 2007, 23 (1), 36-39 (in Chin.).

(21) Jiang, H. Y.; Ji, C.; Cao, Q. E.; Yang, R. Y.; Zhi, H.; Zhang, Y. N. A Study on salt effect on treatment of oily wastewater using microwave radiation. Adv. Mater. Res. 2012, 361-363, 561-564.

(22) Chen, L.; Xie, C.; Zhou, N.; Liu, S.; Wang, Y. Overview of research progress in forward osmosis technology. Water Purif. Technol. 2015, 34 (5), 26-32 (in Chin.).

(23) Petrotos, K. B.; Quantick, P.; Petropakis, H. A study of the direct osmotic concentration of tomato juice in tubular membranemodule configuration. I. The effect of certain basic process parameters on the process performance. J. Membr. Sci. 1998, 150 (1), 99-110.

(24) Holloway, R. W.; Childress, A. E.; Dennett, K. E.; Cath, T. Y. Forward osmosis for concentration of anaerobic digester centrate. Water Res. 2007, 41 (17), 4005-4014.

(25) Yip, N. Y.; Tiraferri, A.; Phillip, W. A.; Schiffman, J. D.; Elimelech, M. High performance thin-film composite forward osmosis membrane. Environ. Sci. Technol. 2010, 44 (10), 3812-3818.

(26) Bell, E. A.; Poynor, T. E.; Newhart, K. B.; Regnery, J.; Coday, B. D.; Cath, T. Y. Produced water treatment using forward osmosis membranes: Evaluation of extended-time performance and fouling. $J$. Membr. Sci. 2017, 525, 77-88.

(27) Li, G.; Wang, J.; Hou, D.; Bai, Y.; Liu, H. Fabrication and performance of PET mesh enhanced cellulose acetate membranes for forward osmosis. J. Environ. Sci. 2016, 45, 7-17.
(28) Yong, J. S. Reverse draw solute transport in forward osmosis systems. Ph.D. Dissertation, Yale University, New Haven, CT, USA, 2012.

(29) Xiao, W.; Guan, P.; Wang, D. Studies on preparation and performance of novel polyamide composite FO membrane. J. Funct. Mater. 2016, 47 (B06), 127-133 (in Chin.).

(30) Xie, M.; Price, W. E.; Nghiem, L. D. Rejection of pharmaceutically active compounds by forward osmosis: Role of solution $\mathrm{pH}$ and membrane orientation. Sep. Purif. Technol. 2012, 93, 107-114 (in Chin.).

(31) Liang, H.; Hung, W.; Yu, H.; Hu, C.; Lee, K.; Lai, J.; Xu, Z. Forward osmosis membranes with unprecedented water flux. J. Membr. Sci. 2017, 529, 47-54.

(32) Elimelech, M.; Zhu, X.; Childress, A. E.; Hong, S. Role of membrane surface morphology in colloidal fouling of cellulose acetate and composite aromatic polyamide reverse osmosis membranes. J. Membr. Sci. 1997, 127 (1), 101-109.

(33) Boussu, K.; Belpaire, A.; Volodin, A.; Van Haesendonck, C.; Van der Meeren, P.; Vandecasteele, C.; Van der Bruggen, B. Influence of membrane and colloid characteristics on fouling of nanofiltration membranes. J. Membr. Sci. 2007, 289, 220-230.

(34) Li, H.; Shi, W.; Du, Q.; Zhou, R.; Zhang, H.; Qin, X. Improved separation and antifouling properties of thin-film composite nanofiltration membrane by the incorporation of cGO. Appl. Surf. Sci. 2017, 407, 260-275.

(35) $\mathrm{Zhu}, \mathrm{Z}$. The relations among hydrophilicity, wettability and contact angle of membranes. Membr. Sci. Technol. 2014, 34 (2), 1-4 (in Chin.).

(36) Dova, M. I.; Petrotos, K. B.; Lazarides, H. N. On the direct osmotic concentration of liquid foods. Part I: Impact of process parameters on process performance. J. Food Eng. 2007, 78 (2), 422430.

(37) Li, G.; Li, X.; Liu, Y.; Wang, D.; He, T.; Gao, C. Forward osmosis and concentration polarization. Prog. Chem. 2010, 22 (5), 812-821 (in Chin.).

(38) Xiao, P.; Li, J.; Ren, Y.; Wang, X. A comprehensive study of factors affecting fouling behavior in forward osmosis. Colloids Surf., $A$ 2016, 499, 163-172.

(39) Coday, B. D.; Almaraz, N.; Cath, T. Y. Forward osmosis desalination of oil and gas wastewater: Impacts of membrane selection and operating conditions on process performance. J. Membr. Sci. 2015, $488,40-55$.

(40) Coday, B. D.; Heil, D. M.; Xu, P.; Cath, T. Y. Effects of transmembrane hydraulic pressure on performance of forward osmosis membranes. Environ. Sci. Technol. 2013, 47, 2386-2393.

(41) Tang, C. Y.; She, Q.; Lay, W. C. L.; Wang, R.; Fane, A. G. Coupled effects of internal concentration polarization and fouling on flux behavior of forward osmosis membranes during humic acid filtration. J. Membr. Sci. 2010, 354 (1), 123-133.

(42) Arena, J. T.; Manickam, S. S.; Reimund, K. K.; Freeman, B. D.; Mccutcheon, J. R. Solute and water transport in forward osmosis using polydopamine modified thin film composite membranes. Desalination 2014, 343, 8-16.

(43) Liu, X.; Wu, J.; Liu, C.; Wang, J. Forward osmosis technology applied for water and wastewater treatment. Acta Sci. Circum. 2016, 36 (8), 2701-2713 (in Chin.).

(44) Bao, X. Prepation of sulfonated polyethersulfone (SPES) thin film composite forward-osmosis membranes and study on its properties. M.S. Thesis, Harbin Institute of Technology, Harbin, China, 2015 (in Chin.).

(45) Gray, G. T.; McCutcheon, J. R.; Elimelech, M. Internal concentration polarization in forward osmosis: role of membrane orientation. Desalination 2006, 197 (1-3), 1-8.

(46) Déon, S.; Dutournié, P.; Fievet, P.; Limousy, L.; Bourseau, P. Concentration polarization phenomenon during the nanofiltration of multi-ionic solutions: Influence of the filtrated solution and operating conditions. Water Res. 2013, 47 (7), 2260-2272.

(47) Déon, S.; Dutournié, P.; Limousy, L.; Bourseau, P. The twodimensional pore and polarization transport model to describe 
mixtures separation by nanofiltration: Model validation. AIChE J. 2011, 57 (4), 985-995.

(48) Gao, Y.; Wang, Y.; Li, W.; Tang, C. Y. Characterization of internal and external concentration polarizations during forward osmosis processes. Desalination 2014, 338, 65-73.

(49) Hancock, N. T.; Cath, T. Y. Solute coupled diffusion in osmotically driven membrane processes. Environ. Sci. Technol. 2009, 43 (17), 6769-6775.

(50) Du, X.; Wang, Y.; Qu, F.; Li, K.; Liu, X.; Wang, Z.; Li, G.; Liang, $\mathrm{H}$. Impact of bubbly flow in feed channel of forward osmosis for wastewater treatment: Flux performance and biofouling. Chem. Eng. J. 2017, 316, 1047-1058.

(51) Zhao, S.; Zou, L.; Tang, C. Y.; Mulcahy, D. Recent developments in forward osmosis: Opportunities and challenges. $J$. Membr. Sci. 2012, 396, 1-21.

(52) Qasim, M.; Darwish, N. A.; Sarp, S.; Hilal, N. Water desalination by forward (direct) osmosis phenomenon: A comprehensive review. Desalination 2015, 374, 47-69.

(53) Zhao, P.; Gao, B.; Yue, Q.; Liu, P.; Shon, H. K. Fatty acid fouling of forward osmosis membrane: Effects of $\mathrm{pH}$, calcium, membrane orientation, initial permeate flux and foulant composition. J. Environ. Sci. 2016, 46, 55-62.

(54) Hawari, A. H.; Kamal, N.; Altaee, A. Combined influence of temperature and flow rate of feeds on the performance of forward osmosis. Desalination 2016, 398, 98-105.

(55) Parida, V.; Ng, H. Y. Forward osmosis organic fouling: Effects of organic loading, calcium and membrane orientation. Desalination 2013, 312, 88-98.

(56) Chun, Y.; Kim, S.; Millar, G. J.; Mulcahy, D.; Kim, I. S.; Zou, L. Forward osmosis as a pre-treatment for treating coal seam gas associated water: Flux and fouling behaviour. Desalination 2017, 403, $144-152$.

(57) Zhao, S.; Zou, L.; Mulcahy, D. Effects of membrane orientation on process performance in forward osmosis applications. J. Membr. Sci. 2011, 382, 308-315.

(58) Lv, L.; Xu, J.; Shan, B.; Gao, C. Concentration performance and cleaning strategy for controlling membrane fouling during forward osmosis concentration of actual oily wastewater. J. Membr. Sci. 2017, $523,15-23$.

(59) Cai, X. Study on high-efficiency water clarifiers for polymer flooding produced water. M.S. Thesis, Jilin University, Jilin, China, 2013 (in Chin.).

(60) Arkhangelsky, E.; Wicaksana, F.; Chou, S.; Al-Rabiah, A. A.; AlZahrani, S. M.; Wang, R. Effects of scaling and cleaning on the performance of forward osmosis hollow fiber membranes. J. Membr. Sci. 2012, 415-416, 101-108.

(61) Liu, S.; Xie, C.; Zhou, N.; Wang, Y. Overview of research progress in membrane fouling and cleaning for forward osmosis membrane. Water Purif. Technol. 2015, 34 (4), 23-30 (in Chin.).

(62) Mi, B.; Elimelech, M. Organic fouling of forward osmosis membranes: Fouling reversibility and cleaning without chemical reagents. J. Membr. Sci. 2010, 348 (1-2), 337-345.

(63) Xue, N.; Pan, T. Research progress on characteization of concentration polarization and membrane fouling of forward osmosis. Membr. Sci. Technol. 2015, 35 (5), 109-113 (in Chin.).

(64) Wang, L.; Huang, D.; Zhou, T.; Zhang, K.; Li, W.; Sun, S.; Wei, J.; Yin, L. Progress on forward osmosis distillation processes for treatment of fracturing flow-back. Petrol. Ind. Appl. 2016, 35 (2), 5-10 (in Chin.). 